\title{
Erratum to: Survival advantage combining a BRAF inhibitor and radiation in BRAF V600E-mutant glioma
}

\author{
Tina Dasgupta ${ }^{1}$ - Aleksandra K. Olow ${ }^{2,3} \cdot$ Xiaodong Yang $^{3} \cdot$ Rintaro Hashizume $^{4}$. \\ Theodore P. Nicolaides ${ }^{5,6} \cdot$ Maxwell Tom $^{5} \cdot$ Yasuyuki Aoki $^{5} \cdot$ Mitchel S. Berger $^{5}$. \\ William A. Weiss ${ }^{5,6,7} \cdot$ Lukas J. A. Stalpers $^{8}$ - Michael Prados ${ }^{5}$ - C. David James ${ }^{4}$. \\ Sabine Mueller ${ }^{6,7} \cdot$ Daphne A. Haas-Kogan ${ }^{3,5}$
}

Published online: 19 January 2016

(C) Springer Science+Business Media New York 2016

\section{Erratum to: J Neurooncol DOI 10.1007/s11060-015-1939-2}

The name of author Theodore P. Nicolaides was omitted in the initial online publication. The online publication has been updated to address this omission. The authors and affiliations listed in this erratum are the same as in the updated article.

The online version of the original article can be found under doi:10.1007/s11060-015-1939-2.

Tina Dasgupta

tinadasgupta2006@gmail.com

1 Valley Radiotherapy Associates, 1500 Rosecrans Avenue, Manhattan Beach, CA 90266, USA

2 Department of Laboratory Medicine, University of California, San Francisco (UCSF), San Francisco, CA 94143, USA

3 Department of Radiation Oncology, University of California, San Francisco (UCSF), San Francisco, CA 94143, USA

4 Department of Neurological Surgery, Northwestern University Feinberg School of Medicine, Chicago, IL 60611, USA

5 Department of Neurological Surgery, University of California, San Francisco (UCSF), San Francisco, CA 94143, USA

6 Department of Pediatrics, University of California, San Francisco, San Francisco, CA 94143, USA

7 Department of Neurology, University of California, San Francisco (UCSF), San Francisco, CA 94143, USA

8 Department of Radiotherapy, Academic Medical Center, University of Amsterdam, Amsterdam, The Netherlands 